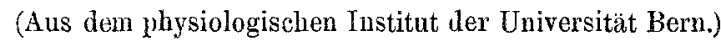

\title{
Die Innervation der Nebenniere durch den Splanchnicus.
}

\author{
Von
}

\section{Leon Asher.}

Die Tatsache, dass die Absonderung von Adrenalin unter dem Einfluss des Nervensystems auf dem Wege des Nervus splanchnicus stehen kann, ist eine durch die Arbeiten von Tscheboksareff, Elli ot, Cannon, Anrep und mir selbst gesicherte. Da der Nachweis dieser Abhängigkeit vom Nervensystem in zwiefacher Richtung von Bedeutung war, einmal, weil dadurch echte innere Sekretion auf physiologisehe Weise unzweifelhaft geworden war, zum zweiten, weil hierdurch die Ausschliesslichkeit der chemischen Regulation der Drüsen mit innerer Sekretion beseitigt worden war, ist es von Bedeutung, dass dieser Nachweis gegen jeden Einwand gefeit sei. Deshalb ist jede Experimentalkritik im Interesse der Sache zu begrüssen. Popielski hat in diesem Archiv (Pflüger's Arch. Bd. 165 S. 565 u. 581. 1916) eine derartige Experimentalkritik versucht, welcher leider entgegengetreten werden muss, weil sie die Tatbestände in nicht zutreffender Weise wiedergibt und dazu geeignet ist, einen wirklich und leicht nachweisbar klaren Sachverhalt zu verdunkeln.

P o pielski's Argumentation läuft darauf hinaus, dass der Übertritt von Adrenalin in die Blutbahn stets beruhe auf einem mechanischen Druck, welcher auf die Nebenniere ausgeübt wird, und er ist bestrebt, allen Arbeiten, in denen gezeigt wurde, dass auf Reizung des Splanchnicus vermehrte Adrenalinabsonderung in das Blut stattfindet, den Versuchsfehler vorzuwerfen, dass mechanischer Druck auf die Nebenniere das Resultat vorgetäuscht habe. Was nun meine eigene Arbeit betrifft (L. Asher, Die innere Seliretion der Nebenniere und deren Innervation. Zeitsehr. f. Biol. Bd. 58 H. 6 S. 286), so ist in derselben die Methodik so genau beschrieben, dass der vollständige Ausschluss des von Popielski vermuteten Versuchsfehlers mit aller wünschenswerten Deutlichkeit daraus hervorgeht. In meinen Versuchen wurde der Nervus splanchnicus in die Gotchschen Kapillarröhrchen zur Reizung von Nerven eingelegt. Diese Gotch'schen Glaselektroden bleiben in der Tiefe der Bauchhöhle liegen, und die Bauchhöhle ist während der ganzen eigentlichen Ver- 
suchsdauer verschlossen. Irgendeine Änderung in der Reizperiode gegenüber der Ruhperiode tritt nicht ein; die einzige Manipulation, welche während der Reizung stattfindet, ist die Öffnung des Vorreiberschlüssels der sekundären Rolle des Induktionsapparates; eine Zerrung an den Elektroden oder irgendeine Druckwirkung auf die Unterleibshöhle findet überhaupt nicht statt, so dasś, wenn es $\mathrm{zu}$ Anzeichen von Adrenalinwirkung in meinen Versuchen gekommen ist, dies ausschliesslich darauf beruht, dass die Reizung des Splanchnicus eben zu einer vermehrten Sekretion von Adrenalin geführt hat. Fast möchte man aus Popielski's Angaben vermuten, dass ihm die einzig zulässige Art der Reizung des Splanchnicus ohne jeden Eingriff am Tiere während der Reizung unbekannt ist. Nicht allein, wenn man die Wirkung auf die Nebenniere studiert, sondern auch bei jeder anderen untersuchten Wirkungsweise dieses Nerven wird man bei der Reizung mechanische Änderungen, wie Zerrung am Splanchnicus und Manipulieren an der Bauchhöhle, vermeiden. Der Vorzug meiner Methode des Nachweises der Adrenalinabsonderung im Untersehied zu manchen anderen Methoden beruht darauf, dass gar keine Eingriffe nötig sind, um Blut zu erhalten, in dem vermehrtes Adrenalin nachzuweisen wäre, sondern sich alles am gleichen Tiere ohne jeden weiteren Eingriff nach den ersten Operationen vollzieht.

Auch im übrigen scheint Popielski meine Methodik missverstanden zu haben. Er schreibt: „Er bedeckte die Nebenniere mit in warmer Kochsalzlösung getränkter Watte unter Vermeidung angeblich jedes Druckes. Dann wandte er aus ganz unverständlichen Gründen Gefässpinzetten zur Abklemmung der Nebennierenvenen an. Die Abklemmung der Nebennierenvene hatte keinen Einfluss auf den Blutdruck ... Es ist offenbar, dass die von Asher angewandten Eingriffe hätten vermieden werden müssen. Gerade sie sind vollkommen ausreichend, um den unbedeutenden Blutdruckanstieg zu erzeugen, welchen der Autor bei Reizung des Splanchnicus beobachtete." Die Bedeckung der Nebenniere mit Watte geschieht während der Operation an der Bauchböhle, um die Nebenniere nicht mit Instrumenten oder mit den Händen zu verletzen. Bei versehlossener Bauchhöhle und der Reizung des Splanchnicus liegen auf der Nebenniere keine drückenden Wattebäuschel. Die Abklemmung der Nebennierenvenen geschah nicht aus unverständlichen Gründen, sondern als Kontrolle, um nach Abschluss der Nebennierenvenen die etwaige Reizwirkung des Nervus splanchnicus zu prüfen. Diese Kontrollabklemmungen geschahen nur in ganz bestimmten Versuchen, und 
zwar am Ende derselben. Die Kontrollversuche haben methodisch mit den Hauptversuchen nichts zu tun. Es bleibt als Endergebnis, dass alle Einwände von Popielski gegen meine Versuchsmethodik auf irrtümlicher Auffassung derselben beruhen, und dass demnach der von mir gefübrte Nachweis der inneren Sekretion der Nebenniere unter dem Einfluss des Nervus splanchnicus zu Recht besteht.

Das gleiche gilt von der schönen Arbeit von T. R. Elliot (T. R. E'lliot, The Control of the suprarenal Glands by the Splanchnic Nerves. Journ. of Physiol. Vol. 44 p. 374. 1912), welche merkwürdigerweise 'von Popielski gar nicht erwähnt wird. Elliot arbeitete an der dezerebrierten Katze, welehe evisceriert worden war, und reizte intrathorakal den Splanchnicus. Demnach war bei dieser Methode während der Reizung gieichfalls die von Popielski vermutete Fehlerquelle ausgeschlossen, und die sehr erhebliche Drucksteigerung, die Elli ot erzielte, beweist in sehr eklatanter Weise die Adrenalinabsonderung ausschliesslich infolge der Reizung des Nervus splanchnicus. Was die Arbeiten von Cannon und von Anrep anlangt, so gehört schon sehr viel Zwang dazu, dieselben im Sinne von Popielski umzudeuten, nachdem die von mir und von Elliot angewandte Methodik die Angelegenheit aus der Sphäre der von Popielski vorgebrachten Vermutungen entrückt hat.

Es soll an dieser Stelle nicht bezweifelt werden, dass mechanischer Druck auf die Nebenniere zu einer Auspressung von Adrenalin führen kann. Übrigens wenn es richtig ist, was Popielski behauptet, dass selbst eine sebr leichte Berührung der Nebenniere zu merklichem Adrenalinaustritt aus derselben führt, so spricht doch die Tatsache sehr zugunsten der Auffassung, dass das Adrenalin teilweise an Orten gelagert ist, von denen her es leicht abgesondert werden kann. Die Frage, ob die rein physiologische Dauerabsonderung der Nebenniere einen Betrag erreicht, dass die bekannten experimeutellen Reaktionen des Adrenalins im Organismus dauernd in geringem Maasse eintreten, bleibe bier unerörtert. Soviel ist jedenfalls durch kritisch einwandfreie Experimentaluntersuchungen festgestellt, dass durch Impulse, welche die Nebenniere auf dem Wege des Nervus splanchnicus erreichen, eine Absonderung von Adrenalin hervorgerufen wird, die sich durch sehr dettliche Reaktionen kund tut, und die eine biologische Aufgabe im Organismus erfült. Popielski's Untersuchungen können nicht das mindeste an der Lehre ändern, dass es eine physiologische innere Sekretion von Adrenalin gibt, und dass dieselbe unter der Herrschaft des Nervensystems steht. 